\title{
Estudio y análisis médico-legal de las muertes en privación de libertad en Málaga (2004-2012)
}

\author{
Study and forensic analysis of deaths in custody in Malaga, \\ Spain (2004-2012)
}

\section{IM. Santos Amaya ${ }^{1}$ \\ F. Martín Cazorla ${ }^{2}$ \\ V. Ramos Medina ${ }^{3}$ \\ LO. Rubio Lamía}

${ }^{1}$ Profesor Titular de Universidad. Área de Medicina Legal y Forense.

Universidad de Málaga

${ }^{2}$ Médico Forense. Servicio de Patología del IML de Málaga

${ }^{3}$ Médico Forense. Jefe de Sección de Histopatología del IML de Málaga

${ }^{4}$ Profesora Doctora Ayudante. Área de Medicina Legal y Forense.

Universidad de Málaga

Correspondencia:

I.M. Santos Amaya

Departamento de Anatomía y Medicina Legal. Facultad de Medicina. Universidad de Málaga Boulevard Louis Pasteur, 32. 29071 Málaga.

E-mail: isantos@uma.es

Fecha de recepción:

04. OCT. 2013

Fecha de aceptación: 18. DIC. 2013

\begin{abstract}
Resumen
Se presenta un estudio sobre todos los casos de muertes en privación de libertad ocurridos en la provincia de Málaga durante los años 2004-2012, que fueron 41 . En cuanto al origen y la causa de la muerte, la mayoría fueron accidentales $(46,3 \%$, incluyendo reacción adversa a drogas), seguidas de suicidios $(24,3 \%)$, muertes de origen natural $(21,9 \%)$ y homicidios (7,3\%). En el estudio se detalla también la edad, el sexo, la nacionalidad, el consumo de tóxicos y el lugar del suceso. Finalmente se exponen cuatro interesantes casos: dos accidentes, un suicidio y un homicidio.
\end{abstract}

Palabras clave: Autopsia. Delirium agitado. Drogas de abuso. Muerte de presos y detenidos. Privación de libertad.

\section{Abstract}

A study of all cases of deaths in custody occurred in the province of Malaga, Spain, during the years 2004 to 2012 is presented. These were 41 . As for the origin and etiology of death, most were accidents $46.3 \%$ (including adverse drug reaction), followed by suicides (24.3\%), natural deaths $(21.9 \%$ ) and homicides $(7.3 \%)$. In the study; age, sex, national consumption of toxic and location of occurrence is detailed. Finally four interesting cases are discussed: two accidents, one suicide and a homicide.

Key words: Agitated delirium. Abuse drugs. Deaths in custody. Freedom deprivation.

\section{Introducción}

Las muertes de personas que se encuentran en situación de privación de libertad, como presos, detenidos y pacientes ingresados involuntariamente en establecimientos psiquiátricos, son conocidas en la literatura médico legal anglosajona como deaths in custody, y han motivado numerosas publicaciones y capítulos exclusivos en los textos más prestigiosos de la especialidad en los últimos años. Palomo Rando et al. ${ }^{1}$ proponen utilizar la denominación de "muerte en privación de libertad" para las muertes que acontecen durante la detención y el enfrentamiento con fuerzas de seguridad, las muertes de internos en centros de detención y prisiones (incluyendo las fugas e intentos de fuga) y las muertes de ingresados de forma involuntaria en centros psiquiátricos.

La muerte en privación de libertad se encuadraría dentro del grupo de muertes sospechosas de crimi- nalidad, lo que a tenor del artículo 343 de la Ley de Enjuiciamiento Criminal sería objeto de la práctica de autopsia y de la correspondiente investigación judicial $^{2}$.

Dentro del grupo de muertes en privación de libertad podemos encontrar diversas situaciones a tener en cuenta: muertes naturales acontecidas en privación de libertad, muertes violentas como suicidio (generalmente ahorcadura), o accidentales como reacción aguda a drogas de abuso o atragantamientos en internos psiquiátricos, y muertes acontecidas durante la detención, como los casos de delirium agitado.

Aunque es evidente que la práctica de la autopsia es necesaria en los apartados segundo y tercero, no es menos cierto que ésta debe realizarse también en los casos que a priori tienen un origen natural pero acontecen en privación de libertad, como pudiera ser un preso que ha sido trasladado al hospital. 
En España no existe oposición legal a que si estas muertes se presentan como de origen natural puedan certificarse e inhumar el cadáver sin más investigaciones. No obstante, en muchos países existe la obligación expresa de practicar la autopsia forense a toda persona muerta en circunstancias relacionadas con el arresto y la privación de libertad. Creemos que las muertes que ocurren en privación de libertad son de los casos más difíciles de resolver por parte del patólogo forense, pues no sólo deberá probar lo que ocurrió sino que también deberá documentar lo que no aconteció, todo ello a la espera de las críticas a que puede verse sometido por parte de familiares y amigos de la víctima, así como de los medios de comunicación.

El objetivo de este trabajo es realizar un análisis descriptivo de todos los casos de muertes en situación de privación de libertad en la provincia de Málaga entre los años 2004 y 2012, periodo que corresponde a la creación y existencia del Instituto de Medicina Legal de Málaga (ILM Málaga, octubre 2003), donde se practican todas las autopsias de la provincia. También se presentan de manera más detallada cuatro interesantes casos de muerte en privación de libertad. Los datos obtenidos pueden servir para conocer la situación, establecer cuáles son las causas más frecuentes de estas muertes y dónde se producen, y proponer medidas para evitarlas.

\section{Material y métodos}

Se incluyen en el estudio los casos médico-legales autopsiados en el Instituto de Medicina Legal de Málaga, entre los años 2004 y 2012, de las muertes bajo custodia, considerando las ocurridas durante los arrestos policiales, en prisión y en la unidad de salud mental por ingreso involuntario.

De un total de 8.555 autopsias realizadas en este periodo, 41 fueron identificadas como muertes en privación de libertad. En el estudio se detalla el origen y la causa de la muerte, la edad, el sexo, la nacionalidad, el consumo de tóxicos y el lugar del suceso.

Se presentan, a modo de ejemplo, cuatro casos interesantes, de diferentes situaciones en que pueden ocurrir muertes en privación de libertad. Estos casos corresponden a una muerte por reacción adversa a drogas en prisión, un suicidio por ahorcamiento en el calabozo policial, una muerte por delirium agitado en la vía pública y un homicidio por compresión toracoabdominal en un hospital psiquiátrico.

\section{Resultados}

El número de muertes en privación de libertad durante el periodo de estudio fue de 41 , lo que representa un $0,47 \%$ de las autopsias realizadas en ese tiempo. La media por año es de 4,5 autopsias, con una variación entre una, en 2004 y 2009, y siete en 2012.

La causa más frecuente fue la accidental $(46,3 \%)$, seguida de los suicidios (24,3\%), las muertes de origen natural $(21,9 \%)$ y los homicidios $(7,3 \%)$. El $75,6 \%$ eran españoles, el $14,6 \%$ de otros países de la Unión Europea y el 9,7\% extracomunitarios. El $87,8 \%$ de los casos eran hombres y sólo el $12,2 \%$ mujeres. La media de edad fue de 44,12 años, siendo el 73,1\% menor de 50 años.

En relación al lugar donde se produjo la muerte, la mayoría aconteció en prisión (41,4\%), seguido de los calabozos de la policía y el hospital psiquiátrico (ambos $21,9 \%$ ), y de otros lugares $(14,6 \%)$, que incluyen dos casos en la vía pública, uno en el coche policial, uno en el juzgado, uno en un centro de internamiento de extranjeros y otro en un hospital de agudos (Tabla 1 ).

\begin{tabular}{cr}
\hline Causa & N \\
\hline Natural & 9 \\
Accidente & 19 \\
Suicidio & 10 \\
Homicidio & 3 \\
Nacionalidad & \\
Española & 31 \\
Unión europea & 6 \\
Extracomunitarios & 4 \\
Edad (años) & \\
$21-30$ & 5 \\
$31-40$ & 11 \\
$41-50$ & 14 \\
$51-60$ & 4 \\
$61-70$ & 5 \\
$71-80$ & 2 \\
Sexo & \\
Hombre & 36 \\
Mujer & 5 \\
Lugar & \\
Comisaria de policía & 9 \\
Prisión & \\
Hospital psiquiátrico & 6 \\
Otros & \\
\hline
\end{tabular}

Tabla 1. Origen y causa de la muerte, nacionalidad, edad, sexo y lugar del fallecimiento. 
De las nueve muertes naturales, el 55,5\% se debieron a cardiopatías, un 33,3\% a tromboembolia pulmonar y una muerte por hemorragia digestiva alta. La media de edad fue de 53,3 años, con una proporción hombre-mujer de 2/1, y la mayoría eran españoles $(77,7 \%)$. Respecto al lugar del fallecimiento, el $66,6 \%$ aconteció en prisión y el 33,3\% en hospital psiquiátrico.

Se produjeron 19 muertes por accidentes, de las cuales el 52,6\% fueron por reacción adversa a drogas, el $26,3 \%$ por delirium agitado, el 10,5\% por sofocación debida a cuerpo extraño, un caso por politraumatismo y otro por intoxicación medicamentosa. La media de edad fue de 40,9 años, la relación hombre-mujer de $8,5 / 1$ y un $84,2 \%$ eran españoles. La muerte ocurrió en prisión en un $42,10 \%$, en comisaría un $21 \%$ y en un hospital psiquiátrico otro $21 \%$, dos casos en la vía pública y uno en el coche policial; estos tres últimos casos correspondieron a muertes por delirium agitado.

De los 10 suicidios registrados, ocho fueron por ahorcadura, uno por precipitación y otro por arma blanca. La media de edad fue de 39,6 años, todos hombres, un $50 \%$ españoles y otro $50 \%$ extranjeros, la mitad de ellos extracomunitarios. Respecto al lugar de la muerte, el $50 \%$ tuvo lugar en comisarías, el $20 \%$ en prisión y el $10 \%$, respectivamente, en los juzgados, en un centro de internamiento de extranjeros y en un hospital de agudos, con un caso en cada uno.

En el periodo estudiado hubo tres homicidios, uno por compresión toracoabdominal, otro por sofocación y otro por politraumatismo. La media de edad fue de 63,6 años y los tres eran hombres españoles. Dos ocurrieron en un hospital psiquiátrico y uno en prisión (Tabla 2).

En cuanto al consumo de alcohol y tóxicos previo al fallecimiento, en un $21,9 \%$ de los casos hubo consumo de alcohol y en un $53,6 \%$ consumo de otros tóxicos; dentro de este último grupo, la droga más consumida fueron las benzodiacepinas $(90,4 \%)$, seguidas por la cocaína (38\%), la metadona (38\%), los opiáceos $(23,8 \%)$ y el cannabis (19\%). En tres casos se produjo consumo de alcohol junto al de otros tóxicos.

Estudiando el lugar donde se produjo la muerte y la causa médico-legal de ésta, encontramos que de los 16 casos ocurridos en prisión el $47 \%$ fueron accidentales (reacción adversa a drogas), el 35,2\% muertes naturales, dos suicidios y un homicidio. De las nueve muertes en comisaría, el 55,5\% fueron suicidios (ahorcadura) y el $44,5 \%$ accidentales. En los hospitales psiquiátricos hubo nueve fallecimientos, el $44,4 \%$ accidentes, el 33,3\% muertes naturales y el $22,2 \%$ homicidios. Respecto a las seis muertes ocurridas en otros lugares, tres fueron suicidios y tres accidentales (delirium agitado).

\section{Caso 1. Muerte por reacción adversa a drogas en prisión}

Varón de 31 años de edad, de nacionalidad española, que en el recuento que realiza el funcionario de prisiones a las $8 \mathrm{~h}$ de la mañana es hallado muerto en decúbito supino en la cama de su celda. Como antecedentes médicos de interés destaca toxicomanía.

En el examen externo se observa hongo de espuma que fluye por los orificios nasales y la boca, erosión frontal, y no se apreciaron signos de venopunción. En el examen interno se observa edema cerebral, equimosis subpleurales y edema pulmonar de gran intensidad (peso de los pulmones $1360 \mathrm{~g}$ ). Las pruebas complementarias detectaron la presencia de cocaína, heroína y benzodiacepinas.

El origen de la muerte fue violento y se determinó como causa inmediata de ella un edema pulmonar tóxico, y como causa fundamental la reacción adversa a drogas de abuso.

\section{Caso 2. Suicidio por ahorcamiento en el calabozo de la policía}

Varón de 50 años de edad, ciudadano extracomunitario, que fue hallado muerto en los calabozos de la policía. Fue arrestado por amenazar a una mujer y trasladado a la comisaría. El sujeto se había colgado del cuello formando el lazo con dos calcetines largos anudados en sus extremos, unidos a su vez a la puerta de la celda usada como punto de suspensión; la ahorcadura era incompleta. A la llegada de los servicios sanitarios se encontraba ya cadáver. No se observaron otros signos de carácter violento.

Una vez en el Servicio de Patología del Instituto de Medicina Legal de Málaga, se realizó una autopsia completa cuyos hallazgos fueron: surco de ahorcadura único, poco profundo, ascendente, que se interrumpe en la región laterocervical izquierda, con erosiones hemorrágicas en el fondo del surco. Además, se observó infiltrado hemorrágico en la musculatura esquelética cervical y fractura incompleta del asta mayor izquierda del hioides y del asta superior izquierda del tiroides. Pulmones congestivos y edematosos. El estudio histopatológico confirmó la vitalidad del surco de ahorcadura. Asimismo, en las muestras remitidas para análisis toxicológico había 2,83 g/l de etanol en sangre. Se estableció que la causa de la muerte se debió a una asfixia mecánica por ahorcadura. 


\begin{tabular}{|c|c|c|c|}
\hline \multicolumn{2}{|l|}{ Muerte natural } & \multicolumn{2}{|l|}{ Suicidios } \\
\hline Edad (años) & $\mathbf{N}$ & Edad (años) & $\mathrm{N}$ \\
\hline $21-30$ & 0 & $21-30$ & 3 \\
\hline $31-40$ & 2 & $31-40$ & 1 \\
\hline $41-50$ & 2 & $41-50$ & 6 \\
\hline $51-60$ & 2 & $51-60$ & 0 \\
\hline $61-70$ & 2 & $61-70$ & 0 \\
\hline $71-80$ & 1 & $71-80$ & 0 \\
\hline \multicolumn{2}{|l|}{ Nacionalidad } & \multicolumn{2}{|l|}{ Nacionalidad } \\
\hline Española & 7 & Española & 5 \\
\hline Unión europea & 1 & Unión europea & 3 \\
\hline Extracomunitarios & 1 & Extracomunitarios & 2 \\
\hline \multicolumn{2}{|l|}{ Sexo } & \multicolumn{2}{|l|}{ Sexo } \\
\hline Hombre & 6 & Hombre & 10 \\
\hline Mujer & 3 & Mujer & 0 \\
\hline \multicolumn{2}{|l|}{ Causa } & \multicolumn{2}{|l|}{ Método } \\
\hline Cardiopatía & 5 & Ahorcadura & 8 \\
\hline Tromboembolia pulmonar & 3 & Precipitación & 1 \\
\hline Hemorragia digestiva alta & 1 & Arma blanca & 1 \\
\hline \multicolumn{2}{|l|}{ Lugar } & \multicolumn{2}{|l|}{ Lugar } \\
\hline Comisaria de policía & 0 & Comisaria de policía & 5 \\
\hline Prisión & 6 & Prisión & 2 \\
\hline Hospital psiquiátrico & 3 & Hospital psiquiátrico & 0 \\
\hline Otros & 0 & Otros & 3 \\
\hline \multicolumn{2}{|l|}{ Accidentes } & \multicolumn{2}{|l|}{ Homicidios } \\
\hline Edad (años) & $\mathbf{N}$ & Edad (años) & $\mathrm{N}$ \\
\hline $21-30$ & 2 & $21-30$ & 0 \\
\hline $31-40$ & 8 & $31-40$ & 0 \\
\hline $41-50$ & 6 & $41-50$ & 0 \\
\hline $51-60$ & 1 & $51-60$ & 1 \\
\hline $61-70$ & 2 & $61-70$ & 1 \\
\hline $71-80$ & 0 & $71-80$ & 1 \\
\hline \multicolumn{2}{|l|}{ Nacionalidad } & \multicolumn{2}{|l|}{ Nacionalidad } \\
\hline Española & 16 & Española & 3 \\
\hline Unión Europea & 2 & Unión Europea & 0 \\
\hline Extracomunitarios & 1 & Extracomunitarios & 0 \\
\hline \multicolumn{2}{|l|}{ Sexo } & \multicolumn{2}{|l|}{ Sexo } \\
\hline Hombre & 17 & Hombre & 3 \\
\hline Mujer & 2 & Mujer & 0 \\
\hline \multicolumn{2}{|l|}{ Método } & \multicolumn{2}{|l|}{ Método } \\
\hline RAPSUSI & 10 & Compresión toracoabdominal & 1 \\
\hline Delirium agitado & 5 & Sofocación & 1 \\
\hline Sofocación por cuerpo extraño & 2 & Politraumatismo & 1 \\
\hline Politraumatismo & 1 & & \\
\hline Intoxicación medicamentosa & 1 & & \\
\hline \multicolumn{2}{|l|}{ Lugar } & \multicolumn{2}{|l|}{ Lugar } \\
\hline Comisaria de policía & 4 & Comisaria de policía & 0 \\
\hline Prisión & 8 & Prisión & 1 \\
\hline Hospital psiquiátrico & 4 & Hospital psiquiátrico & 2 \\
\hline Otros & 3 & Otros & 0 \\
\hline
\end{tabular}

Tabla 2.

Origen y causa de la muerte, nacionalidad, edad, sexo, método y lugar del fallecimiento. 


\section{Caso 3. Muerte por delirium agitado en la vía pública}

Varón de 42 años de edad, ciudadano británico, que falleció poco después de ser retenido por miembros de las fuerzas de seguridad. El motivo de la detención fue su agresividad y comportamiento extraño en la vía pública, que requirió la intervención de varios agentes para reducirlo.

El cadáver tenía un índice de masa corporal (IMC) de 38,6 , y en el examen externo se observaron múltiples lesiones consistentes en erosiones y contusiones. En el examen interno se hallaron tres fracturas costales, ausencia de lesiones en órganos vitales y signos inespecíficos de asfixia. El estudio toxicológico reveló la presencia en sangre de cocaína y sus metabolitos, lo que indicaba un consumo reciente. Se concluyó como causa de la muerte un delirium agitado.

\section{Caso 4. Homicidio por compresión toracoabdominal en un hospital psiquiátrico}

Varón de 61 años de edad, español, que fue encontrado cadáver a las 22:30 h en la habitación del hospital psiquiátrico donde se encontraba ingresado. Como antecedentes personales figuraba oligofrenia y psicosis en tratamiento con Risperdal ${ }^{\circledR}$, Luminal ${ }^{\circledR}$ y Etumina ${ }^{\circledR}$. El compañero de habitación declaró a la policía haberse sentado encima del fallecido durante un tiempo.

Una vez trasladado el cadáver al Instituto de Medicina Legal de Málaga se procede a la realización de la autopsia, en la que se observó la presencia de "mascarilla equimótica" en la cabeza y el cuello (congestión), diversas lesiones de tipo equimosis y erosiones en el tórax, el abdomen y los miembros superiores e inferiores (leves signos de violencia, algunos de ellos compatibles con compresión torácica), y signos inespecíficos de asfixia. En el estudio histopatológico se descartó la presencia de patología orgánica que justificase la muerte del sujeto. El estudio toxicológico fue negativo. El origen de la muerte fue violento y se determinó como causa la compresión toracoabdominal.

\section{Discusión}

Nuestros resultados de 4,5 muertes por año son ligeramente inferiores a los publicados por Okoye et $a .^{3}$ en su estudio sobre la muerte en custodia en el Estado de Nebraska (Estados Unidos), de 5,4 muertes por año entre 2003-2009. En relación con los datos epidemiológicos en España, no existe un registro oficial de muertes en privación de libertad; sólo se dispone de las muertes que tienen lugar en centros penitenciarios. Así, el último informe general realizado por la Dirección General de Instituciones Penitenciarias ${ }^{4}$, dependiente del Ministerio del Interior, es del año 2009 y se notificaron 224 muertes en centros penitenciarios, con una tasa de mortalidad de 3,42 por mil internos (3,70 en 2008). Las causas de mortalidad fueron: naturales distintas al virus de la inmunodeficiencia humana (VIH) 57,1\%, VIH $7,6 \%$, drogas $21 \%$, suicidio $12,1 \%$, accidental $0,9 \%$ y agresión $1,3 \%$.

En cuanto a la etiología médico-legal, en nuestro estudio predominan los accidentes al incluir en ellos las reacciones adversas a drogas. Debemos tener en cuenta que, de los 16 casos ocurridos en prisión, casi la mitad fueron por esta causa. Según Instituciones Penitenciarias, en el año 2009 fueron 47 los fallecidos por drogas (57 en 2008), con una tasa de 0,72 por mil internos (0,93 en el año 2008). En el análisis histórico de las muertes en custodia realizado por Grant et al. ${ }^{5}$ sobre casos de la oficina del Medical Examiner de Maryland (1939-2004), la intoxicación por drogas también fue mayoritaria en las prisiones. Destacamos que en estos 9 años se han registrado cinco casos de muerte por delirum agitado (dos en la vía pública y uno, respectivamente, en el coche policial, la comisaría de policía y el hospital psiquiátrico).

Respecto a los suicidios, al igual que en otros estudios, la mayoría fue por ahorcadura (ocho frente a dos por otros métodos). Resulta de interés destacar que más de la mitad ocurrieron en comisarías de policía y un $50 \%$ eran sujetos extranjeros. En prisión se produjeron dos suicidios. En el año 2009, el número de suicidios en los centros penitenciarios fue de 27 , con una tasa de incidencia de 0,41 por mil internos (0,32 por mil internos en 2008). Estas tasas, según Instituciones Penitenciarias, aunque sensiblemente inferiores a las de la Unión Europea, son superiores a las de la población general para iguales grupo de edad y sexo. En el trabajo de Okoye et al. ${ }^{3}$ sobre muertes en custodia en el Estado de Nebraska, entre 2003 y 2009 , los suicidios representan el $21 \%$ de las muertes en custodia, cifra ligeramente inferior a la obtenida por nosotros (24,3\%).

En nuestro estudio, las muertes naturales representan el $21,9 \%$, cifra más baja que la obtenida por Okoye et al. ${ }^{3}(36 \%)$, aunque superior a la hallada por Grant et al. ${ }^{5}$ en Maryland entre 1990 y 2000 (10\%), aunque en este último trabajo hay que tener presente que 
el $63,5 \%$ de las muertes fueron calificadas como indeterminadas. Según Instituciones Penitenciarias, el número de internos fallecidos por causa natural no VIH en el año 2009 fue de 128 , con una tasa de 1,9 por cada mil; la mayoría mientras estaban ingresados en centros hospitalarios, y en prisión fallecieron el $41,4 \%$. Las tres principales causas fueron (como en años anteriores) la cardiopatía isquémica, los tumores (con predominio del carcinoma de pulmón) y la hepatopatía crónica. Así pues, resulta lógico que en nuestro estudio, teniendo en cuenta que en España puede certificarse la defunción por causa natural de un interno, resulte menor el número y mayoritariamente más comunes las causas de muerte súbita del adulto como cardiopatías y la tromboembolia pulmonar.

En nuestro trabajo se recogen tres homicidios, que suponen el $7,3 \%$ de las muertes en privación de libertad. En el estudio de Grant et al. ${ }^{5}$ los homicidios representaron un $8,5 \%$, y en el de Okoye et al. ${ }^{3}$ un $21 \%$. Según Instituciones Penitenciarias, la mortalidad por agresión en 2009 fue de tres internos, el $1,3 \%$ de las muertes en prisión, con una tasa de 0,05 por mil internos; la media de los fallecidos por esta causa en los últimos 10 años es de 2,3 internos por año. En nuestro medio, los homicidios fueron infrecuentes y se produjeron en instituciones cerradas, la mayoría en un hospital psiquiátrico, y la principal causa de la muerte fue la asfixia.

En relación con el lugar del fallecimiento, encontramos que existe una relación entre la causa de la muerte y el tipo de institución donde acontece. Así, las muertes de origen natural fueron más frecuentes en prisión y en instituciones de salud mental, la reacción adversa a drogas en prisión, los suicidios en los calabozos de comisarías y los homicidios en el hospital psiquiátrico.

\section{Caso 1. Muerte por reacción adversa a drogas en prisión}

El Observatorio Español sobre Drogas define el indicador de mortalidad por Reacción Aguda a Sustancias Psicoactivas (muerte RASUPSI) como el número de muertes por reacción adversa aguda tras el consumo intencional y no médico de sustancias psicoactivas en personas entre 10 y 64 años de edad. El indicador de mortalidad en España se puso en marcha en 1989, coordinado por el Plan Nacional sobre Drogas, inicialmente en seis ciudades, y posteriormente se ha ido ampliando a muchas zonas del territorio nacional. El conocimiento de este indicador es importante para diseñar políticas y programas destinados a reducir los problemas relacionados con las drogas.
Según el Observatorio Europeo de las Drogas y las Toxicomanías, las cifras de muertes relacionadas con drogas de abuso son muy diversas en los distintos países y oscilan entre 3 y 85 muertes por millón habitantes; fue la causa del $4 \%$ de todas las muertes de los europeos entre los 15 y los 39 años de edad, afectando fundamentalmente a los hombres y con una media de edad de 35 años ${ }^{6}$.

En nuestro estudio, el $80 \%$ de las muertes por reacción adversa a drogas se produjo en prisión, un $10 \%$ en el hospital psiquiátrico y otro $10 \%$ en la comisaría de policía. Los estudios toxicológicos realizados revelaron policonsumo en el $80 \%$ de los casos, lo cual está acorde con los diferentes estudios que indican que una proporción considerable de las muertes inducidas por drogas pueden estar relacionadas con el policonsumo. Las principales drogas detectadas fueron metadona (70\%), cocaína (40\%) y heroína (40\%). Según el Boletín Epidemiológico de la Dirección General de Instituciones Penitenciarias ${ }^{4}$, sus resultados son concordantes con los nuestros en cuanto a que la metadona es la sustancia más detectada, seguida de la cocaína. La mayor presencia de metadona en el ámbito penitenciario puede explicarse por la existencia de programas de mantenimiento con metadona para los internos. Sería necesario mejorar los protocolos de seguridad en las prisiones para evitar la entrada y la distribución de drogas de abuso.

\section{Caso 2. Suicidio por ahorcamiento en el calabozo de la policía}

En las muertes en custodia, gran cantidad de casos suceden por suicidio, en comisaría y en sujetos extranjeros. La literatura científica recoge que el $70 \%$ al $80 \%$ de los individuos hablaron de ello antes de hacerlo ${ }^{7}$. En nuestro caso, éste no verbalizó en ningún momento los deseos de suicidio.

El suicidio suele ocurrir durante las primeras 72 horas del ingreso en comisaría (frecuentemente en las primeras 24 horas). Se observa que a pesar de mantener las pautas de prevención de suicidio (retirada de objetos peligrosos o cinturón), los sujetos siguen cometiéndolo y con frecuencia utilizando la ropa que portan. En este caso, el sujeto lo hizo con sus propios calcetines.

En nuestra opinión, sería conveniente una revisión del protocolo de actuación y prevención en sujetos que son detenidos y llevados a comisaría, sobre todo extranjeros. 


\section{Caso 3. Muerte por delirium agitado en la vía pública}

El presente caso es un cuadro propio de personas que consumen tóxicos, fundamentalmente cocaína, lo cual desencadena un trastorno mental con un comportamiento extraño y extremadamente violento, que lleva aparejado casi siempre la intervención de la policía, que ante esa actitud violenta y desafiante procede a su detención empleando la fuerza necesaria para contrarrestar tal situación. Por ello, estas muertes pueden encuadrarse dentro de lo que en la literatura médico-legal se conoce como muertes en privación de libertad (death in custody).

Aunque las muertes en custodia policial son un raro suceso durante un arresto, los casos de esta naturaleza despiertan una gran atención por parte de los medios de comunicación, generan en la comunidad un malestar hacia los cuerpos y fuerzas de seguridad del estado y normalmente originan litigios penales y civiles, alegando una muerte injusta. Identificar la causa de la muerte supone un importante desafío para el médico forense, porque a menudo hay múltiples factores que pueden haber contribuido a ella. Con frecuencia los hallazgos de la autopsia no revelan alteraciones anatómicas que puedan explicar la muerte. En estos casos, el médico forense debe identificar los hechos que proporcionen una gran probabilidad para establecer la causa de la muerte. El cuadro clínico se caracteriza por hipertermia, agitación psicomotriz y muerte.

La muerte en el síndrome de delirium agitado resulta de una arritmia cardiaca fatal debido a un estado hiperadrenérgico causado a su vez por los siguientes mecanismos: el forcejeo y la agitación que acontecen en el delirium agitado producen una estimulación del sistema nervioso simpático, una disminución brusca en las concentraciones séricas de potasio tras el cese del forcejeo y un incremento en las concentraciones de catecolaminas ${ }^{8}$.

El estado hiperadrenérgico se ve invariablemente agravado por los efectos de drogas estimulantes; por medicación que ejerce los mismos efectos que los estimulantes, elevando las concentraciones de catecolaminas o prolongando el intervalo QT; o por la existencia de una enfermedad natural que por sí misma es insuficiente para causar la muerte, pero que en combinación con el estado hiperadrenérgico podría explicarla9.

Los factores de riesgo en el síndrome de delirium agitado son el sexo masculino, la patología cardíaca preexistente, la obesidad (IMC >25), la contención mecánica del detenido que dificulte los movimientos respiratorios (asfixia posicional) y el consumo de drogas (especialmente cocaína). En nuestro estudio se recogen cinco casos de muerte por síndrome de delirium agitado, todos de ellos varones, lo cual se corresponde con los diferentes estudios realizados. En tres de ellos se detectó cocaína en sangre en el momento de la muerte, lo cual es similar a los resultados de los estudios que presentan a esta droga como el tóxico más frecuentemente detectado en este cuadro, aunque también pueden observarse otros. De los cincos casos, cuatro estaban bajo custodia policial y el quinto ingresado en un centro psiquiátrico de manera involuntaria. Tres de ellos murieron en la vía pública, otro en los calabozos de la policía y otro en el hospital. En el caso que se detalla puede apreciarse que el paciente presenta todos los factores considerados de riesgo antes expuestos. Es muy necesario que las fuerzas de seguridad conozcan la existencia de este cuadro de delirio agitado y se les enseñe cómo actuar en consecuencia ${ }^{10}$.

\section{Caso 4. Homicidio por compresión toracoabdominal en un hospital psiquiátrico}

Es interesante que, en nuestro estudio, entre las muertes en privación de libertad que ocurren en hospitales psiquiátricos se observen dos casos de homicidio: uno ya descrito (muerte por compresión toracoabdominal) y otro por sofocación con una almohada.

Los homicidios no son frecuentes en los hospitales psiquiátricos, pero sin duda podría mejorarse su prevención detectando y aislando en estancias diferentes a pacientes que se consideren potencialmente agresivos.

\section{Conclusiones}

Nuestras cifras de muertes en privación de libertad son semejantes a la que se presentan en otros estudios recogidos en la literatura científica.

Es necesario un registro único de las muertes en privación de libertad en España, y no sólo de los fallecimientos en prisión que recoge Instituciones Penitenciarias en la actualidad. Podría establecerse un observatorio, tal como se hizo respecto a las muertes por reacción aguda a sustancias psicoactivas, a partir de los datos de las autopsias forenses realizadas en los institutos de medicina legal en España. 
En relación con las muertes por reacción adversa a drogas, estimamos necesario mejorar los protocolos de seguridad en las prisiones para evitar la entrada, la distribución y el abuso de drogas en ellas.

En nuestra opinión, también sería conveniente revisar el protocolo de actuación y prevención del suicidio en los sujetos que son detenidos y llevados a los calabozos de las comisarías, sobre todo si se trata de ciudadanos extranjeros.

Es muy prioritario que las fuerzas de seguridad conozcan la existencia del cuadro de delirio agitado y se les enseñe cómo actuar en consecuencia, para evitar muertes en los arrestos.

Aunque los homicidios en los hospitales psiquiátricos no son frecuentes, sin duda podría mejorarse su prevención detectando y aislando en estancias diferentes a los pacientes mientras presenten un cuadro de heteroagresividad.

Los autores declaran no tener conflicto de intereses.

\section{Bibliografía}

1. Palomo Rando JL, Ramos Medina V, Santos Amaya IM. Muerte en privación de libertad (MPL). Cuadernos de Medicina Forense. 2004;35(1):37- 50.

2. Ley de Enjuiciamiento Criminal. Real decreto de 14 de septiembre de 1882 por el que se aprueba la Ley de Enjuiciamiento Criminal. Disponible en: https://www.boe.es/diario_boe/txt.php?id=BOEA-1882-6036

3. Okoye CN, Okoye M, Lynch, DT. An analysis and report of custodial deaths in Nebraska, USA: part II. Journal of Forensisc and Legal Medicine. 2012; 19:465-9.

4. Mortalidad en Instituciones Penitenciarias. Boletín Epidemiológico de Instituciones Penitenciarias. 2009;16(1-2-3). Editado el 10-5-2011.

5. Grant JR, Southall PE, Fowler DR, Mealey J, Thomas EJ, Kinlock T. Death in custody: a historical analysis. Journal of Forensic Science. 2007;52(5):1177-81.

6. Delgado Bueno S, Bandrés Moya F, Lucena Romero J. Tratado de medicina legal y ciencias forenses.
En: Delgado Bueno S, Bandrés Moya F, Lucena Romero J. Muerte por reacción adversa al consumo de drogas de abuso (RADA): a) Cocaína. Muerte súbita asociada al consumo de cocaína. Delirium excitado. b) Drogas de síntesis. c) Opiáceos. Barcelona: ed. Bosch, 2011; Tomo III. Cap. 156: 637-57.

7. McCleave NR, Latham D. Self-injorious behaviour in police custody. Journal of Clinical Forensic Medicine. 1998;5:13-6.

8. Young DB, Srivastava TN, Fitzovich DE, Kivlighn SD, Hamaguchi M. Potassium and catecholamine concentrations in the immediate post exercise period. Am J Med Sci. 1992;304:150-3.

9. Di Maio T, di Maio V. Excited delirium syndrome: cause of death and prevention. 1st ed. Taylor \& Francis. Boca Raton, FL. USA. 2006.

10. Martín Cazorla F, Santos Amaya IM, Ramos Medina V, Rubio Lamia LO, Palomo Rando JL. Muerte por síndrome de delirium agitado en Andalucía. Rev Esp Med L. 2010;36(2):62-7. 